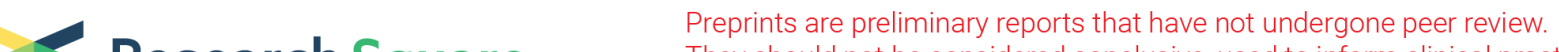 Research Square They should not be considered conclusive, used to inform clinical practice, or referenced by the media as validated information.
}

\section{Modest therapeutic effects of low-frequency transcutaneous electric nerve stimulation on insomnia among older adults: A 4-week multi-center, randomized controlled study}

Hyuk joo Lee

Department of Psychiatry, Uijeongbu Eulji Medical Center, Eulji University, Gyeonggi

Jung Kyung Hong

Department of Psychiatry, Seoul National University Bundang Hospital, Seongnam

Hayun Choi

Department of Psychiatry, Veteran Health Service Medical Center, Seoul

\section{Seockhoon Chung}

Department of Psychiatry, Asan Medical Center, University of Ulsan College of Medicine, Seoul

In-Young Yoon ( $D$ iyoon@snu.ac.kr)

Department of Psychiatry, Seoul National University Bundang Hospital, Seongnam

\section{Research Article}

Keywords: LF-TENS, PSQI score, blood cortisol level, experimental and control groups

Posted Date: March 30th, 2021

DOl: https://doi.org/10.21203/rs.3.rs-363345/v1

License: (우 (i) This work is licensed under a Creative Commons Attribution 4.0 International License. Read Full License 


\section{Abstract}

A 4-week, multi-center, randomized controlled study was conducted to evaluate the therapeutic efficacy and safety of low-frequency transcutaneous electric nerve stimulation (LF-TENS) for insomnia disorder. A total of 160 individuals aged 40 to 80 years with insomnia disorder were included and randomized to the experimental group receiving active device $(n=81)$ or control group receiving sham device $(n=79)$. Both groups used the device for four weeks, more than five days a week. The participants also completed preand post-intervention assessment with questionnaires, sleep diaries, wrist actigraphy, and blood tests. We found a significant improvement of subjective sleep quality, depressive/anxiety symptoms, and blood cortisol level in both experimental and control groups. Notably, among patients aged over 60 years, the experimental group showed better improvement after intervention in the change of PSQI score $(2.60 \pm 0.46$ vs. $1.22 \pm 0.46, p=0.039$; Cohen's $d=0.99$ vs 0.45$)$ and blood cortisol level $(-1.97 \pm 0.53 \mu \mathrm{g} / \mathrm{dl}$ vs. $-0.16 \pm$ $0.53 \mu \mathrm{g} / \mathrm{dl}, \mathrm{p}=0.007$; Cohen's $d=0.56$ vs 0.05 ). We found that LF-TENS was effective in improving subjective sleep quality in older adults aged over 60 years. The hypothalamic-pituitary-adrenal axis might be related to the therapeutic mechanism.

\section{Introduction}

Insomnia disorder is the most common sleep disorder characterized by dissatisfaction with sleep quality or quantity that causes considerable daytime functional impairment. Its prevalence has been estimated ranging from 10 to 33 percent in adults according to the reports of various studies conducted worldwide ${ }^{1-}$ ${ }^{4}$. Moreover, a longitudinal study revealed that $46 \%$ of insomniacs complained of persistent insomnia symptoms even after three years, suggestive of chronicity of the disease ${ }^{5}$. Insomnia disorder is also associated with physical and mental health problems such as cardiovascular diseases ${ }^{6}$, depressive disorder ${ }^{7}$, and cognitive impairment ${ }^{8}$.

The currently established treatment for insomnia disorder is restricted to short-term pharmacotherapy and cognitive behavior therapy (CBT), along with modification of precipitating and perpetuating factors for insomnia ${ }^{9}$. However, these conventional therapies have some limitations. The most widely used medications for insomnia including benzodiazepine receptor agonists have potential risk of side effects, such as dependency, tolerance ${ }^{10}$ and cognitive decline ${ }^{11}$. CBT is costly and time-consuming because regular in-person encounters with therapists and good adherence of patients to the treatment sessions are required. Furthermore, over $40 \%$ of patients could not achieve remission with combined pharmacotherapy and $\mathrm{CBT}$ for six months ${ }^{12}$. To overcome these shortcomings of conventional therapies and to achieve better treatment outcomes, clinicians have tried to find an alternative therapeutic approach for insomnia.

Cranial electrotherapy stimulation (CES) is an electrical neurostimulation technique that delivers a pulsed, alternating low-intensity electric current across the head via electrodes on the earlobes or scalp. It was first introduced as a novel therapeutic modality for insomnia in the 1970s and since then, a couple of studies have indicated that CES could alleviate insomnia symptoms and improve sleep quality ${ }^{13-15}$. Meanwhile, some other researches have failed to confirm the therapeutic effects of CES on insomnia ${ }^{16,17}$ and a recent 
systematic review also revealed inconclusive results regarding the efficacy of CES for insomnia treatment 18.

Transcutaneous electric nerve stimulation (TENS) is a different type of neurostimulation modality which applies electric current pulses by electrodes through peripheral skin surface other than the head. TENS was found to be effective for pain control in various pain conditions ${ }^{19}$ and it is widely used for relieving neuromuscular pain with only minor adverse effects, including mild erythema and itching sensations underneath the electrodes ${ }^{20}$. In particular, low-frequency $(<1000 \mathrm{~Hz})$ TENS (LF-TENS) is effective in local stimulation of the application site without cardiovascular side effects compared to high-frequency TENS (HF-TENS) ${ }^{21}$. Considering that chronic insomnia disorder is associated with inappropriate physiological hyperarousal ${ }^{22}$ and that the possible analgesic mechanisms of LF-TENS include the activation of gammaaminobutyric acid (GABA) pathway ${ }^{19}$, LF-TENS could be helpful for treating sleep disturbances. However, to our best knowledge, only few studies has examined the effects of LF-TENS on sleep ${ }^{23,24}$ and no randomized controlled trial on this issue has been conducted.

To investigate safety and therapeutic effects of LF-TENS on insomnia, we have performed an open trial with 40 patients suffering from chronic insomnia. Our data demonstrated that four weeks intervention of LF-TENS can improve subjective sleep quality without intolerable or persistent adverse reactions ${ }^{24}$. However, the methodological shortcomings of this pilot study such as the absence of control group and objective sleep measurements may limit the interpretation of results. Hence, in the present study, we aimed to verify the safety and efficacy of LF-TENS application for the management of insomnia disorder, using a randomized double-blind and placebo-controlled parallel study design.

\section{Results}

\section{Baseline demographic and clinical characteristics}

A total of 196 patients suffering from insomnia disorder were enrolled in the study and 176 patients were randomized. Of those, 90 and 86 persons had been assigned to the experimental group and the control group, respectively. The dropout rate was $10.0 \%(9 / 90)$ in the experimental group and $8.1 \%(7 / 86)$ in the control group, with no significant difference between the two groups. Finally, 160 patients completed all the study protocols and were included for the analysis (Fig. 1). When we compared the baseline demographic and clinical characteristics including objective sleep data from the PSG records, no statistically significant difference was found between the experimental and control group except for BMI and periodic limb movement index (PLMI). Compared to the control group, the experimental group showed significantly lower $\mathrm{BMI}$ and higher PLMI at the baseline assessment (Table 1). In addition, we found no significant difference in all baseline characteristics between those participants completed the study protocol and those who dropped out (data not shown). 
Table 1

Baseline demographic and clinical characteristics of the whole study population

\begin{tabular}{|c|c|c|c|}
\hline Characteristics & Experimental group $(\mathrm{N}=\mathbf{8 1})$ & Control group $(\mathrm{N}=79)$ & $\mathbf{P}$ \\
\hline Sex, male & $29(35.8)$ & $31(39.6)$ & 0.653 \\
\hline Age, years & $60.81 \pm 9.26$ & $59.62 \pm 8.69$ & 0.402 \\
\hline $\mathrm{BMI}, \mathrm{kg} / \mathrm{m}^{2}$ & $22.22 \pm 2.51$ & $23.16 \pm 2.42$ & 0.018 \\
\hline Medical illness, presence & $42(51.9)$ & $42(53.2)$ & 0.868 \\
\hline Medication use, presence & $45(55.6)$ & $47(59.5)$ & 0.614 \\
\hline \multicolumn{4}{|l|}{ Questionnaires, score } \\
\hline PSQI & $12.47 \pm 2.72$ & $11.91 \pm 2.65$ & 0.191 \\
\hline ESS & $7.52 \pm 4.73$ & $8.47 \pm 5.27$ & 0.232 \\
\hline BDI & $15.84 \pm 9.37$ & $15.34 \pm 8.28$ & 0.723 \\
\hline BAI & $11.51 \pm 7.68$ & $11.16 \pm 9.70$ & 0.805 \\
\hline NRS & $3.10 \pm 2.43$ & $3.06 \pm 2.51$ & 0.928 \\
\hline \multicolumn{4}{|l|}{ Polysomnography } \\
\hline Time in bed, min & $483.71 \pm 25.90$ & $486.16 \pm 24.12$ & 0.537 \\
\hline Sleep latency, min & $26.32 \pm 29.74$ & $24.66 \pm 29.90$ & 0.726 \\
\hline Wake after sleep onset, min & $108.93 \pm 55.27$ & $104.63 \pm 51.75$ & 0.613 \\
\hline Total sleep time, min & $348.50 \pm 56.42$ & $356.87 \pm 58.37$ & 0.358 \\
\hline Sleep efficiency, \% & $72.16 \pm 11.52$ & $73.00 \pm 14.02$ & 0.682 \\
\hline AHI, events/hr & $5.06 \pm 4.44$ & $5.52 \pm 4.21$ & 0.499 \\
\hline PLMI, events/hr & $12.98 \pm 19.79$ & $6.45 \pm 13.80$ & 0.017 \\
\hline \multicolumn{4}{|c|}{$\begin{array}{l}\text { Data are presented as mean } \pm \text { standard deviation for numerical data and number (percentage) for } \\
\text { categorized data. }\end{array}$} \\
\hline \multicolumn{4}{|c|}{ Independent t-test and chi-square test were performed to examine inter-group differences } \\
\hline \multicolumn{4}{|c|}{$\begin{array}{l}\text { BMI, Body mass index; PSQI, Pittsburgh sleep quality index; ISI, Insomnia severity scale; ESS, Epworth } \\
\text { sleepiness scale; BDI, Beck's depression inventory; BAI, Beck's anxiety inventory; NRS, numeric rating } \\
\text { scale; AHI, apnea-hypopnea index; PLMI, periodic limb movement index. }\end{array}$} \\
\hline
\end{tabular}

\section{Safety reports}

Tolerability data were available for 175 participants because one participant had dropped out before the first assessment for adverse events. Adverse reactions were reported by 35 patients (20\%) at least once during the treatment period; headache $(n=7)$, chest discomfort $(n=2)$, myalgia $(n=5)$ skin rash $(n=3)$, 
urticaria $(n=1)$, itching $(n=9)$, tingling sensation $(n=6)$ dizziness $(n=3)$, epigastric pain $(n=2)$, nausea ( $n$ $=1$ ) and fatigue $(n=3)$. The symptoms obviously not related to the device use, such as common cold, gastrointestinal disturbances due to food intake, and pain induced by other underlying medical conditions, were not regarded as adverse reactions. Three patients had dropped out due to adverse events; two in the experimental group (tingling sensation and back pain) and one in the control group (shoulder pain). All the adverse reactions were tolerable and self-limiting within several days for remaining participants. Also, no significant difference was found in the occurrence of adverse events between completers and drop-outs [19.4\% (31/160) vs. 26.7\% (4/15), p = 0.500]. Furthermore, among the completers, the incidence rate of adverse events did not differ significantly between the experimental and control group in the incidence rate of adverse events [17.3\% (14/81) vs. $21.5 \%(17 / 79), p=0.498]$.

\section{Effects of LF-TENS intervention}

There was no substantial difference in the number of device use ( $24.05 \pm 3.19$ days vs. $23.94 \pm 2.66$ days, $p$ $=0.809)$ and the duration of daily use (53.74 \pm 6.12 minutes vs. $54.50 \pm 5.88$ minutes, $p=0.422)$ between the experimental group and the control group. The average current intensity of LF-TENS was $1.12 \pm 0.13$ $\mathrm{mA}$ at the neck and $1.13 \pm 0.13 \mathrm{~mA}$ at shoulder. Regarding rescue medication administration, those who used the active device showed slightly lower proportion of zolpidem use [35.9\% (28/81) vs. 43.4\% (34/79), $p=0.272$ ] and lower dosage of zolpidem (9.51 $\pm 16.82 \mathrm{mg}$ vs. $14.37 \pm 20.28 \mathrm{mg}, p=0.100)$, although statistically not significant.

We tested the changes in the outcome measures before and after intervention period in the experimental and control groups among the whole study population. After four weeks of intervention, significant reductions of the PSQI, BDI, and BAI score were found in both study arms. Although the amplitude of these improvements was greater in the experimental group, it did not reach statistical significance (Table 3$)$. The positive treatment response rate was 37.0\% (30/81) in the experimental group and 30.4\% (24/79) in the control group, yielding no substantial difference between the two groups ( $p=0.373$ ) (Fig. 2A). As for sleep parameters, both the experimental and control groups have reported an evident improvement in all the subjective sleep quality based on sleep diaries, without distinct between-group difference. In blood test results, meaningful differences before and after treatment were observed in serum cortisol levels both in the experimental and control groups, producing no significant difference in the changes of serum cortisol levels between the two groups. There was no profound change in actigraphy after study protocol in both study groups, without any noticeable between-group difference. 
Table 2

The comparison of outcome measures before and after 4 weeks of intervention among participants $>60$ years of age

\begin{tabular}{|c|c|c|c|c|c|c|c|}
\hline \multirow[t]{2}{*}{ Characteristics } & \multicolumn{3}{|c|}{ Experimental group $(\mathrm{N}=40)$} & \multicolumn{3}{|c|}{ Control group $(\mathrm{N}=41)$} & \multirow[t]{2}{*}{$\mathrm{P}^{\ddagger}$} \\
\hline & $\begin{array}{l}\text { Pre- } \\
\text { intervention }\end{array}$ & $\begin{array}{l}\text { Post- } \\
\text { intervention }\end{array}$ & $P^{+}$ & $\begin{array}{l}\text { Pre- } \\
\text { intervention }\end{array}$ & $\begin{array}{l}\text { Post- } \\
\text { intervention }\end{array}$ & $P^{+}$ & \\
\hline \multicolumn{8}{|l|}{$\begin{array}{l}\text { Questionnaires, } \\
\text { score }\end{array}$} \\
\hline PSQI & $\begin{array}{l}13.00 \pm \\
2.21\end{array}$ & $\begin{array}{l}10.37 \pm \\
3.04\end{array}$ & $\begin{array}{l}< \\
0.001\end{array}$ & $\begin{array}{l}12.15 \pm \\
2.59\end{array}$ & $\begin{array}{l}10.95 \pm \\
2.70\end{array}$ & 0.002 & 0.039 \\
\hline ESS & $7.80 \pm 4.54$ & $6.78 \pm 4.19$ & 0.124 & $7.51 \pm 5.34$ & $6.85 \pm 4.50$ & 0.250 & 0.957 \\
\hline BDI & $\begin{array}{l}17.90 \pm \\
11.03\end{array}$ & $\begin{array}{l}14.25 \pm \\
8.53\end{array}$ & 0.006 & $\begin{array}{l}15.68 \pm \\
9.36\end{array}$ & $\begin{array}{l}14.00 \pm \\
8.37\end{array}$ & 0.029 & 0.287 \\
\hline BAl & $\begin{array}{l}11.82 \pm \\
7.73\end{array}$ & $9.72 \pm 6.50$ & 0.002 & $\begin{array}{l}11.34 \pm \\
11.26\end{array}$ & $\begin{array}{l}9.56 \pm \\
10.15\end{array}$ & 0.048 & 0.903 \\
\hline NRS & $3.05 \pm 2.56$ & $2.68 \pm 2.33$ & 0.223 & $3.10 \pm 2.60$ & $2.78 \pm 2.48$ & 0.358 & 0.789 \\
\hline Sleep diary & $N=34$ & & & $N=34$ & & & \\
\hline $\mathrm{SL}$, min & $\begin{array}{l}65.17 \pm \\
41.44\end{array}$ & $\begin{array}{l}37.60 \pm \\
30.65\end{array}$ & $<.001$ & $\begin{array}{l}57.92 \pm \\
41.12\end{array}$ & $\begin{array}{l}44.92 \pm \\
37.03\end{array}$ & 0.028 & 0.127 \\
\hline WASO, min & $\begin{array}{l}98.18 \pm \\
70.67\end{array}$ & $\begin{array}{l}57.07 \pm \\
59.72\end{array}$ & $<.001$ & $\begin{array}{l}91.36 \pm \\
64.08\end{array}$ & $\begin{array}{l}69.46 \pm \\
63.35\end{array}$ & 0.013 & 0.128 \\
\hline TST, min & $\begin{array}{l}249.90 \pm \\
78.81\end{array}$ & $\begin{array}{l}324.32 \pm \\
72.85\end{array}$ & $<.001$ & $\begin{array}{l}254.13 \pm \\
72.26\end{array}$ & $\begin{array}{l}303.98 \pm \\
73.53\end{array}$ & 0.001 & 0.247 \\
\hline $\mathrm{SE}, \%$ & $\begin{array}{l}61.50 \pm \\
17.21\end{array}$ & $\begin{array}{l}78.32 \pm \\
14.76\end{array}$ & $\begin{array}{l}< \\
0.001\end{array}$ & $\begin{array}{l}64.01 \pm \\
17.88\end{array}$ & $\begin{array}{l}74.09 \pm \\
17.72\end{array}$ & 0.001 & 0.073 \\
\hline Actigraphy & $N=35$ & & & $N=35$ & & & \\
\hline $\mathrm{SL}$, min & $6.30 \pm 7.04$ & $7.87 \pm 8.42$ & 0.402 & $5.73 \pm 5.51$ & $8.30 \pm 8.87$ & 0.179 & 0.677 \\
\hline WASO, min & $\begin{array}{l}85.21 \pm \\
43.51\end{array}$ & $\begin{array}{l}88.54 \pm \\
49.15\end{array}$ & 0.688 & $\begin{array}{l}72.02 \pm \\
33.92\end{array}$ & $\begin{array}{l}74.87 \pm \\
30.82\end{array}$ & 0.448 & 0.884 \\
\hline
\end{tabular}

Data are presented as mean \pm standard deviation.

${ }^{\dagger}$ Paired t-test was performed to examine within-group differences

‡ Analysis of covariance was performed to examine between-group differences after controlling for age, sex, and baseline BMI.

PSQI, Pittsburgh sleep quality index; ESS, Epworth sleepiness scale; BDI, Beck's depression inventory; BAl, Beck's anxiety inventory; NRS, numeric rating scale; SL, sleep latency; WASO, wake after sleep onset; TST, total sleep time; SE, sleep efficiency; IL-6, interleukin-6; CRP, C-reactive protein; TNF-alpha, tumor necrosis factor-alpha. 


\begin{tabular}{|c|c|c|c|c|c|c|c|}
\hline \multirow[t]{2}{*}{ Characteristics } & \multicolumn{3}{|c|}{ Experimental group $(\mathrm{N}=40)$} & \multicolumn{3}{|c|}{ Control group $(N=41)$} & \multirow[t]{2}{*}{$\mathrm{P}^{\ddagger}$} \\
\hline & $\begin{array}{l}\text { Pre- } \\
\text { intervention }\end{array}$ & $\begin{array}{l}\text { Post- } \\
\text { intervention }\end{array}$ & $P^{+}$ & $\begin{array}{l}\text { Pre- } \\
\text { intervention }\end{array}$ & $\begin{array}{l}\text { Post- } \\
\text { intervention }\end{array}$ & $P^{+}$ & \\
\hline TST, min & $\begin{array}{l}332.29 \pm \\
56.17\end{array}$ & $\begin{array}{l}327.36 \pm \\
55.71\end{array}$ & 0.471 & $\begin{array}{l}326.36 \pm \\
67.07\end{array}$ & $\begin{array}{l}331.04 \pm \\
64.83\end{array}$ & 0.565 & 0.656 \\
\hline $\mathrm{SE}, \%$ & $\begin{array}{l}78.80 \pm \\
8.47\end{array}$ & $\begin{array}{l}78.03 \pm \\
9.82\end{array}$ & 0.590 & $\begin{array}{l}80.86 \pm \\
7.44\end{array}$ & $\begin{array}{l}79.82 \pm \\
7.32\end{array}$ & 0.236 & 0.584 \\
\hline \multicolumn{8}{|l|}{ Blood test } \\
\hline IL-6, pg/ml & $2.00 \pm 2.91$ & $1.49 \pm 1.36$ & 0.334 & $1.57 \pm 1.53$ & $1.99 \pm 2.84$ & 0.267 & 0.282 \\
\hline $\begin{array}{l}\text { TNF-alpha, } \\
\mathrm{pg} / \mathrm{ml}\end{array}$ & $0.92 \pm 0.32$ & $0.85 \pm 0.31$ & 0.073 & $0.82 \pm 0.28$ & $0.88 \pm 0.39$ & 0.131 & 0.007 \\
\hline $\begin{array}{l}\text { Glucose, } \\
\mathrm{mg} / \mathrm{dL}\end{array}$ & $\begin{array}{l}102.55 \pm \\
31.77\end{array}$ & $\begin{array}{l}98.88 \pm \\
20.69\end{array}$ & 0.343 & $\begin{array}{l}97.27 \pm \\
13.19\end{array}$ & $\begin{array}{l}95.00 \pm \\
11.93\end{array}$ & 0.171 & 0.698 \\
\hline CRP, mg/L & $\begin{array}{l}3.84 \pm \\
17.74\end{array}$ & $0.62 \pm 0.60$ & 0.258 & $1.70 \pm 5.44$ & $1.41 \pm 3.99$ & 0.401 & 0.320 \\
\hline Cortisol, $\mu \mathrm{g} / \mathrm{dl}$ & $\begin{array}{l}10.51 \pm \\
2.90\end{array}$ & $8.87 \pm 2.94$ & 0.004 & $\begin{array}{l}10.58 \pm \\
2.66\end{array}$ & $\begin{array}{l}10.42 \pm \\
3.52\end{array}$ & 0.775 & 0.007 \\
\hline Insulin, mIU/L & $6.39 \pm 8.35$ & $6.96 \pm 9.85$ & 0.793 & $4.55 \pm 2.23$ & $4.97 \pm 3.46$ & 0.374 & 0.897 \\
\hline \multicolumn{8}{|c|}{ Data are presented as mean \pm standard deviation. } \\
\hline \multicolumn{8}{|c|}{${ }^{\dagger}$ Paired t-test was performed to examine within-group differences } \\
\hline \multicolumn{8}{|c|}{$\begin{array}{l}\text { ¥ Analysis of covariance was performed to examine between-group differences after controlling for age, } \\
\text { sex, and baseline BMI. }\end{array}$} \\
\hline \multicolumn{8}{|c|}{$\begin{array}{l}\text { PSQI, Pittsburgh sleep quality index; ESS, Epworth sleepiness scale; BDI, Beck's depression inventory; } \\
\text { BAI, Beck's anxiety inventory; NRS, numeric rating scale; SL, sleep latency; WASO, wake after sleep } \\
\text { onset; TST, total sleep time; SE, sleep efficiency; IL-6, interleukin-6; CRP, C-reactive protein; TNF-alpha, } \\
\text { tumor necrosis factor-alpha. }\end{array}$} \\
\hline
\end{tabular}


Table 3

The comparison of outcome measures before and after 4 weeks of intervention among the whole participants

\begin{tabular}{|c|c|c|c|c|c|c|c|}
\hline \multirow[t]{2}{*}{ Characteristics } & \multicolumn{3}{|c|}{ Experimental group $(\mathrm{N}=81)$} & \multicolumn{3}{|c|}{ Control group $(\mathrm{N}=79)$} & \multirow[t]{2}{*}{$\mathrm{P}^{\mathrm{b}}$} \\
\hline & $\begin{array}{l}\text { Pre- } \\
\text { intervention }\end{array}$ & $\begin{array}{l}\text { Post- } \\
\text { intervention }\end{array}$ & $P^{a}$ & $\begin{array}{l}\text { Pre- } \\
\text { intervention }\end{array}$ & $\begin{array}{l}\text { Post- } \\
\text { intervention }\end{array}$ & $P^{a}$ & \\
\hline \multicolumn{8}{|l|}{$\begin{array}{l}\text { Questionnaires, } \\
\text { score }\end{array}$} \\
\hline PSQI & $\begin{array}{l}12.47 \pm \\
2.72\end{array}$ & $\begin{array}{l}10.30 \pm \\
3.36\end{array}$ & $<.001$ & $\begin{array}{l}11.91 \pm \\
2.65\end{array}$ & $\begin{array}{l}10.44 \pm \\
3.09\end{array}$ & $<.001$ & 0.118 \\
\hline ESS & $7.52 \pm 4.73$ & $7.16 \pm 4.31$ & 0.457 & $8.47 \pm 5.27$ & $7.63 \pm 4.40$ & 0.101 & 0.442 \\
\hline BDI & $\begin{array}{l}15.84 \pm \\
9.37\end{array}$ & $\begin{array}{l}13.06 \pm \\
8.31\end{array}$ & $<.001$ & $\begin{array}{l}15.34 \pm \\
8.28\end{array}$ & $\begin{array}{l}13.77 \pm \\
7.91\end{array}$ & 0.006 & 0.263 \\
\hline BAl & $\begin{array}{l}11.51 \pm \\
7.68\end{array}$ & $9.48 \pm 7.28$ & $<_{0.001}$ & $\begin{array}{l}11.16 \pm \\
9.70\end{array}$ & $9.22 \pm 8.65$ & $\hat{0}_{0.001}$ & 1.000 \\
\hline NRS & $3.10 \pm 2.42$ & $2.79 \pm 2.32$ & 0.176 & $3.06 \pm 2.51$ & $2.71 \pm 2.37$ & 0.181 & 0.917 \\
\hline Sleep diary & $N=71$ & & & $N=66$ & & & \\
\hline $\mathrm{SL}$, min & $\begin{array}{l}64.26 \pm \\
41.86\end{array}$ & $\begin{array}{l}39.85 \pm \\
30.82\end{array}$ & $<_{0.001}$ & $\begin{array}{l}56.53 \pm \\
38.39\end{array}$ & $\begin{array}{l}42.88 \pm \\
40.68\end{array}$ & 0.004 & 0.138 \\
\hline WASO, min & $\begin{array}{l}94.53 \pm \\
73.71\end{array}$ & $\begin{array}{l}59.98 \pm \\
53.94\end{array}$ & $<.001$ & $\begin{array}{l}93.60 \pm \\
81.18\end{array}$ & $\begin{array}{l}67.37 \pm \\
69.40\end{array}$ & $\begin{array}{l}< \\
0.001\end{array}$ & 0.383 \\
\hline TST, min & $\begin{array}{l}258.39 \pm \\
81.59\end{array}$ & $\begin{array}{l}315.51 \pm \\
67.70\end{array}$ & $<.001$ & $\begin{array}{l}258.49 \pm \\
77.85\end{array}$ & $\begin{array}{l}304.82 \pm \\
76.96\end{array}$ & $<.001$ & 0.637 \\
\hline SE, \% & $\begin{array}{l}62.68 \pm \\
17.90\end{array}$ & $\begin{array}{l}76.62 \pm \\
14.76\end{array}$ & $<.001$ & $\begin{array}{l}64.56 \pm \\
18.91\end{array}$ & $\begin{array}{l}74.68 \pm \\
17.98\end{array}$ & $<.001$ & 0.221 \\
\hline Actigraphy & $N=72$ & & & $N=69$ & & & \\
\hline $\mathrm{SL}$, min & $5.83 \pm 6.13$ & $6.71 \pm 7.03$ & 0.410 & $6.79 \pm 8.62$ & $7.51 \pm 8.71$ & 0.654 & 0.957 \\
\hline WASO, min & $\begin{array}{l}76.54 \pm \\
40.76\end{array}$ & $\begin{array}{l}78.15 \pm \\
43.67\end{array}$ & 0.742 & $\begin{array}{l}69.55 \pm \\
32.90\end{array}$ & $\begin{array}{l}69.26 \pm \\
33.44\end{array}$ & 0.924 & 0.957 \\
\hline
\end{tabular}

Data are presented as mean \pm standard deviation.

a Paired t-test was performed to examine within-group differences

${ }^{b}$ Analysis of covariance was performed to examine between-group differences after controlling for age, sex, and baseline BMI.

PSQI, Pittsburgh sleep quality index; ESS, Epworth sleepiness scale; BDI, Beck's depression inventory; BAl, Beck's anxiety inventory; NRS, numeric rating scale; SL, sleep latency; WASO, wake after sleep onset; TST, total sleep time; SE, sleep efficiency; IL-6, interleukin-6; CRP, C-reactive protein; TNF-alpha, tumor necrosis factor-alpha. 


\begin{tabular}{|c|c|c|c|c|c|c|c|}
\hline \multirow[t]{2}{*}{ Characteristics } & \multicolumn{3}{|c|}{ Experimental group $(\mathrm{N}=81)$} & \multicolumn{3}{|c|}{ Control group $(\mathrm{N}=79)$} & \multirow[t]{2}{*}{$\mathrm{P}^{\mathrm{b}}$} \\
\hline & $\begin{array}{l}\text { Pre- } \\
\text { intervention }\end{array}$ & $\begin{array}{l}\text { Post- } \\
\text { intervention }\end{array}$ & $\mathbf{P}^{a}$ & $\begin{array}{l}\text { Pre- } \\
\text { intervention }\end{array}$ & $\begin{array}{l}\text { Post- } \\
\text { intervention }\end{array}$ & $P^{a}$ & \\
\hline TST, min & $\begin{array}{l}337.74 \pm \\
57.49\end{array}$ & $\begin{array}{l}331.31 \pm \\
55.35\end{array}$ & 0.217 & $\begin{array}{l}330.14 \pm \\
78.32\end{array}$ & $\begin{array}{l}336.00 \pm \\
61.08\end{array}$ & 0.480 & 0.299 \\
\hline $\mathrm{SE}, \%$ & $\begin{array}{l}80.82 \pm \\
7.90\end{array}$ & $\begin{array}{l}80.19 \pm \\
9.10\end{array}$ & 0.506 & $\begin{array}{l}81.62 \pm \\
7.63\end{array}$ & $\begin{array}{l}81.68 \pm \\
7.58\end{array}$ & 0.925 & 0.847 \\
\hline \multicolumn{8}{|l|}{ Blood test } \\
\hline IL-6, pg/ml & $2.10 \pm 3.74$ & $1.73 \pm 3.10$ & 0.214 & $1.53 \pm 1.40$ & $2.62 \pm 8.33$ & 0.246 & 0.140 \\
\hline $\begin{array}{l}\text { TNF-alpha, } \\
\mathrm{pg} / \mathrm{ml}\end{array}$ & $0.83 \pm 0.30$ & $0.80 \pm 0.30$ & 0.170 & $0.80 \pm 0.29$ & $0.82 \pm 0.33$ & 0.453 & 0.121 \\
\hline $\begin{array}{l}\text { Glucose, } \\
\mathrm{mg} / \mathrm{dL}\end{array}$ & $\begin{array}{l}96.21 \pm \\
23.79\end{array}$ & $\begin{array}{l}95.25 \pm \\
16.64\end{array}$ & 0.631 & $\begin{array}{l}95.91 \pm \\
14.92\end{array}$ & $\begin{array}{l}96.49 \pm \\
17.27\end{array}$ & 0.655 & 0.647 \\
\hline CRP, mg/L & $\begin{array}{l}2.14 \pm \\
12.51\end{array}$ & $0.70 \pm 1.16$ & 0.306 & $1.18 \pm 3.96$ & $1.46 \pm 4.64$ & 0.528 & 0.248 \\
\hline Cortisol, $\mu \mathrm{g} / \mathrm{dl}$ & $\begin{array}{l}10.70 \pm \\
3.03\end{array}$ & $9.66 \pm 3.93$ & 0.020 & $\begin{array}{l}11.03 \pm \\
3.29\end{array}$ & $\begin{array}{l}10.07 \pm \\
3.63\end{array}$ & 0.034 & 0.484 \\
\hline Insulin, mIU/L & $5.20 \pm 6.15$ & $6.16 \pm 8.05$ & 0.395 & $4.67 \pm 2.53$ & $5.83 \pm 4.97$ & 0.029 & 0.978 \\
\hline \multicolumn{8}{|c|}{ Data are presented as mean \pm standard deviation. } \\
\hline \multicolumn{8}{|c|}{ a Paired t-test was performed to examine within-group differences } \\
\hline \multicolumn{8}{|c|}{$\begin{array}{l}\mathrm{b} \text { Analysis of covariance was performed to examine between-group differences after controlling for age, } \\
\text { sex, and baseline BMI. }\end{array}$} \\
\hline \multicolumn{8}{|c|}{$\begin{array}{l}\text { PSQI, Pittsburgh sleep quality index; ESS, Epworth sleepiness scale; BDI, Beck's depression inventory; } \\
\text { BAI, Beck's anxiety inventory; NRS, numeric rating scale; SL, sleep latency; WASO, wake after sleep } \\
\text { onset; TST, total sleep time; SE, sleep efficiency; IL-6, interleukin-6; CRP, C-reactive protein; TNF-alpha, } \\
\text { tumor necrosis factor-alpha. }\end{array}$} \\
\hline
\end{tabular}

Table 2 describes the effects of LF-TENS in elderly patients over 60 years of age. We found a more prominent decrease of PSQI score in the experimental group (13.00 \pm 2.21 to $10.37 \pm 3.04)$ compared to the control group ( $12.15 \pm 2.59$ to $10.95 \pm 2.70)$, after adjusting for possible confounders $(p=0.039)$. Estimated effect size was much larger in the experimental group than in the control group (Cohen's $d=0.99$ vs 0.45 ). The rate of positive treatment response was also higher in the experimental group compared to those of the control group [42.5\% (17/40) vs. $22.0 \%$ (9/41), $p=0.048$ ] (Fig. 2B). Figure 3 represents the changes in PSQI score after intervention for each participant. Regarding blood test results, serum cortisol levels were significantly decreased in the experimental group $(10.51 \pm 2.90 \mu \mathrm{g} / \mathrm{dl}$ to $8.87 \pm 2.94 \mu \mathrm{g} / \mathrm{dl}, \mathrm{p}=0.004)$ but not in the control group $(10.58 \pm 2.66 \mu \mathrm{g} / \mathrm{dl}$ to $10.42 \pm 3.52 \mu \mathrm{g} / \mathrm{dl}, \mathrm{p}=0.775)$, which also resulted in a substantial difference in the decrease of cortisol concentration between the two group $(p=0.007)$. Although statistically insignificant, we found a trend-level decrease of serum TNF-alpha levels in the experimental 
group $(0.92 \pm 0.32 \mathrm{pg} / \mathrm{ml}$ to $0.85 \pm 0.31 \mathrm{pg} / \mathrm{ml}, \mathrm{p}=0.073)$, while an increase was observed in the control group $(0.82 \pm 0.28 \mathrm{pg} / \mathrm{ml}$ to $0.88 \pm 0.39 \mathrm{pg} / \mathrm{ml}, \mathrm{p}=0.131)$. Also, a significant inter-group difference was found between the two groups in the change of serum TNF-alpha concentration $(p=0.007)$. Compared to the control group, the experimental group showed larger estimated effect size in the serum cortisol (Cohen's $d=0.56$ vs 0.05 ) and TNF-alpha levels (Cohen's $d=0.22$ vs -0.18 ).

Among the middle-aged patients aged 40 to 60 years, we could not find a statistically meaningful difference in the changes of outcome measures between the experimental and control groups (Table 4). In addition, no evident difference was observed both in the positive treatment response rate across the two groups [31.7\% (13/41) vs. 39.5\% (15/38), p = 0.471] (Fig. 2C). 
Table 4

The comparison of outcome measures before and after 4 weeks of intervention among middle-aged participants aged 40 to 60 years

\begin{tabular}{|c|c|c|c|c|c|c|c|}
\hline \multirow[t]{2}{*}{ Characteristics } & \multicolumn{3}{|c|}{ Experimental group $(\mathrm{N}=41)$} & \multicolumn{3}{|c|}{ Control group $(\mathrm{N}=38)$} & \multirow[t]{2}{*}{$\mathrm{P}^{\mathrm{b}}$} \\
\hline & $\begin{array}{l}\text { Pre- } \\
\text { intervention }\end{array}$ & $\begin{array}{l}\text { Post- } \\
\text { intervention }\end{array}$ & $P^{a}$ & $\begin{array}{l}\text { Pre- } \\
\text { intervention }\end{array}$ & $\begin{array}{l}\text { Post- } \\
\text { intervention }\end{array}$ & $\mathrm{P}^{\mathrm{a}}$ & \\
\hline \multicolumn{8}{|l|}{$\begin{array}{l}\text { Questionnaires, } \\
\text { score }\end{array}$} \\
\hline PSQI & $\begin{array}{l}11.95 \pm \\
3.07\end{array}$ & $\begin{array}{l}10.22 \pm \\
3.69\end{array}$ & 0.001 & $\begin{array}{l}11.66 \pm \\
2.72\end{array}$ & $9.89 \pm 3.42$ & 0.001 & 0.888 \\
\hline ESS & $7.24 \pm 4.95$ & $7.54 \pm 4.45$ & 0.675 & $9.50 \pm 5.07$ & $8.47 \pm 4.18$ & 0.239 & 0.343 \\
\hline BDI & $\begin{array}{l}13.83 \pm \\
6.97\end{array}$ & $\begin{array}{l}11.90 \pm \\
8.02\end{array}$ & 0.044 & $\begin{array}{l}14.97 \pm \\
7.04\end{array}$ & $\begin{array}{l}13.53 \pm \\
7.48\end{array}$ & 0.097 & 0.723 \\
\hline BAl & $\begin{array}{l}11.20 \pm \\
7.71\end{array}$ & $9.24 \pm 8.05$ & 0.017 & $\begin{array}{l}10.97 \pm \\
7.81\end{array}$ & $8.84 \pm 6.79$ & 0.003 & 0.942 \\
\hline NRS & $3.15 \pm 2.33$ & $2.90 \pm 2.43$ & 0.475 & $3.03 \pm 2.46$ & $2.63 \pm 2.27$ & 0.339 & 0.865 \\
\hline Sleep diary & $N=37$ & & & $N=32$ & & & \\
\hline $\mathrm{SL}$, min & $\begin{array}{l}63.42 \pm \\
42.79\end{array}$ & $\begin{array}{l}41.92 \pm \\
31.25\end{array}$ & 0.004 & $\begin{array}{l}55.06 \pm \\
35.87\end{array}$ & $\begin{array}{l}40.71 \pm \\
44.73\end{array}$ & 0.058 & 0.734 \\
\hline WASO, min & $\begin{array}{l}91.18 \pm \\
77.21\end{array}$ & $\begin{array}{l}62.65 \pm \\
48.71\end{array}$ & 0.004 & $\begin{array}{l}95.98 \pm \\
97.14\end{array}$ & $\begin{array}{l}65.16 \pm \\
76.27\end{array}$ & 0.018 & 0.619 \\
\hline TST, min & $\begin{array}{l}266.19 \pm \\
84.38\end{array}$ & $\begin{array}{l}307.42 \pm \\
62.51\end{array}$ & 0.002 & $\begin{array}{l}263.13 \pm \\
84.29\end{array}$ & $\begin{array}{l}305.72 \pm \\
81.61\end{array}$ & 0.010 & 0.591 \\
\hline $\mathrm{SE}, \%$ & $\begin{array}{l}63.76 \pm \\
18.69\end{array}$ & $\begin{array}{l}75.06 \pm \\
14.78\end{array}$ & $<.001$ & $\begin{array}{l}65.14 \pm \\
20.22\end{array}$ & $\begin{array}{l}75.30 \pm \\
18.52\end{array}$ & 0.004 & 0.866 \\
\hline Actigraphy & $N=37$ & & & $N=34$ & & & \\
\hline $\mathrm{SL}$, min & $5.39 \pm 5.18$ & $5.61 \pm 5.30$ & 0.841 & $\begin{array}{l}7.87 \pm \\
10.93\end{array}$ & $6.50 \pm 8.57$ & 0.500 & 0.631 \\
\hline WASO, min & $\begin{array}{l}68.35 \pm \\
36.68\end{array}$ & $\begin{array}{l}68.3 \pm \\
35.73\end{array}$ & 0.996 & $\begin{array}{l}67.00 \pm \\
32.12\end{array}$ & $\begin{array}{l}63.48 \pm \\
35.46\end{array}$ & 0.455 & 0.972 \\
\hline
\end{tabular}

Data are presented as mean \pm standard deviation.

a Paired t-test was performed to examine within-group differences

${ }^{b}$ Analysis of covariance was performed to examine between-group differences after controlling for age, sex, and baseline BMI

PSQI, Pittsburgh sleep quality index; ESS, Epworth sleepiness scale; BDI, Beck's depression inventory; BAI, Beck's anxiety inventory; NRS, numeric rating scale; SL, sleep latency; WASO, wake after sleep onset; TST, total sleep time; SE, sleep efficiency; IL-6, interleukin-6; CRP, C-reactive protein; TNF-alpha, tumor necrosis factor-alpha. 


\begin{tabular}{|c|c|c|c|c|c|c|c|}
\hline \multirow[t]{2}{*}{ Characteristics } & \multicolumn{3}{|c|}{ Experimental group $(\mathrm{N}=41)$} & \multicolumn{3}{|c|}{ Control group $(\mathrm{N}=38)$} & \multirow[t]{2}{*}{$\mathrm{P}^{\mathrm{b}}$} \\
\hline & $\begin{array}{l}\text { Pre- } \\
\text { intervention }\end{array}$ & $\begin{array}{l}\text { Post- } \\
\text { intervention }\end{array}$ & $\mathbf{P}^{a}$ & $\begin{array}{l}\text { Pre- } \\
\text { intervention }\end{array}$ & $\begin{array}{l}\text { Post- } \\
\text { intervention }\end{array}$ & $P^{a}$ & \\
\hline TST, min & $\begin{array}{l}342.90 \pm \\
59.01\end{array}$ & $\begin{array}{l}335.04 \pm \\
55.51\end{array}$ & 0.322 & $\begin{array}{l}334.02 \pm \\
89.29\end{array}$ & $\begin{array}{l}341.09 \pm \\
57.48\end{array}$ & 0.833 & 0.627 \\
\hline $\mathrm{SE}, \%$ & $\begin{array}{l}82.74 \pm \\
6.90\end{array}$ & $\begin{array}{l}82.24 \pm \\
7.96\end{array}$ & 0.697 & $\begin{array}{l}82.39 \pm \\
7.85\end{array}$ & $\begin{array}{l}83.60 \pm \\
7.46\end{array}$ & 0.304 & 0.726 \\
\hline \multicolumn{8}{|l|}{ Blood test } \\
\hline IL-6, pg/ml & $2.19 \pm 4.44$ & $1.96 \pm 4.16$ & 0.127 & $1.53 \pm 1.26$ & $\begin{array}{l}3.30 \pm \\
11.69\end{array}$ & 0.357 & 0.262 \\
\hline $\begin{array}{l}\text { TNF-alpha, } \\
\mathrm{pg} / \mathrm{ml}\end{array}$ & $0.75 \pm 0.27$ & $0.76 \pm 0.28$ & 0.652 & $0.79 \pm 0.30$ & $0.76 \pm 0.23$ & 0.434 & 0.262 \\
\hline $\begin{array}{l}\text { Glucose, } \\
\mathrm{mg} / \mathrm{dL}\end{array}$ & $\begin{array}{l}90.02 \pm \\
8.27\end{array}$ & $\begin{array}{l}91.71 \pm \\
10.51\end{array}$ & 0.179 & $\begin{array}{l}94.45 \pm \\
16.64\end{array}$ & $\begin{array}{l}98.11 \pm \\
21.68\end{array}$ & 0.068 & 0.455 \\
\hline CRP, mg/L & $0.48 \pm 0.51$ & $0.77 \pm 1.53$ & 0.194 & $0.61 \pm 0.61$ & $1.53 \pm 5.31$ & 0.293 & 0.564 \\
\hline Cortisol, $\mu \mathrm{g} / \mathrm{dl}$ & $\begin{array}{l}10.89 \pm \\
3.18\end{array}$ & $\begin{array}{l}10.43 \pm \\
4.61\end{array}$ & 0.508 & $\begin{array}{l}11.52 \pm \\
3.83\end{array}$ & $9.69 \pm 3.75$ & 0.012 & 0.316 \\
\hline Insulin, mIU/L & $4.04 \pm 2.21$ & $5.38 \pm 5.81$ & 0.087 & $4.81 \pm 2.85$ & $6.77 \pm 6.11$ & 0.047 & 0.625 \\
\hline \multicolumn{8}{|c|}{ Data are presented as mean \pm standard deviation. } \\
\hline \multicolumn{8}{|c|}{ a Paired t-test was performed to examine within-group differences } \\
\hline \multicolumn{8}{|c|}{$\begin{array}{l}{ }^{b} \text { Analysis of covariance was performed to examine between-group differences after controlling for age, } \\
\text { sex, and baseline BMI }\end{array}$} \\
\hline \multicolumn{8}{|c|}{$\begin{array}{l}\text { PSQI, Pittsburgh sleep quality index; ESS, Epworth sleepiness scale; BDI, Beck's depression inventory; } \\
\text { BAI, Beck's anxiety inventory; NRS, numeric rating scale; SL, sleep latency; WASO, wake after sleep } \\
\text { onset; TST, total sleep time; SE, sleep efficiency; IL-6, interleukin-6; CRP, C-reactive protein; TNF-alpha, } \\
\text { tumor necrosis factor-alpha. }\end{array}$} \\
\hline
\end{tabular}

\section{Discussion}

This study demonstrated the safety and therapeutic effect of low-frequency electrical stimulation for elderly insomnia patients without comorbid medical or psychiatric conditions or certain sleep disorders. We found a greater improvement in subjective sleep quality and a higher treatment response rate in those with LF-TENS treatment compared to those with sham treatment, in older insomnia patients over 60 years of age. In line with this finding, among the elderly patients, a significant decrease in serum cortisol level was observed only in the experimental group. With regard to safety issues, no serious adverse reactions had occurred during the study period and the risk of adverse events of LF-TENS treatment did not differ from that of sham treatment. 
Our findings demonstrated the efficacy and safety of electrical stimulation therapy targeting on the peripheral muscle, without direct stimulating effect on central nervous system (CNS) activity. In this study, bilateral upper trapezius muscles were selected as application sites for several reasons. Since the trapezius muscles are innervated by spinal accessory nerve and cervical nerves $\mathrm{C} 2$ to $\mathrm{C} 4{ }^{36}$, the therapeutic effects of LF-TENS on trapezius muscles may affect CNS via this neuronal pathway. This muscle is also known to be related to sleep disturbances. A prospective study reported that an increased muscle response in the upper trapezius muscle could be a strong predictor of sleep complaints ${ }^{37}$. In addition, chronic trapezius myalgia might be associated with anxiety and depression ${ }^{38}$, which may contribute to the aggravation of insomnia symptoms. Moreover, in consistent with our findings, our preliminary study revealed the therapeutic effects of LF-TENS administration on the trapezius muscles without crucial adverse reactions ${ }^{24}$.

The exact mechanism behind the effects of LF-TENS on insomnia still remains unclear yet. One possible explanation could be that well-established analgesic effects of LF-TENS ${ }^{19,20}$ may help ameliorate insomnia symptoms. Chronic pain is thought to be closely related with sleep disturbances via hyperarousal following the experience of pain 22 and proper management of chronic pain is important for the management of insomnia symptoms ${ }^{39,40}$. In the current study, however, no significant post-intervention change of pain intensity was observed for both between experimental and control groups in the elderly as well as in the middle-aged. Additional analysis yielded the similar results after exclusion of patients with adverse events that can affect the pain intensity (data not shown). Our pilot study also revealed no significant difference in pain severity before and after LF-TENS ${ }^{24}$. These findings may, to some extent, result from the low baseline pain severity in our study participants since we have excluded those with medical or psychiatric comorbidities that can be accompanied by severe pain. Our results also suggest that the therapeutic efficacy of LF-TENS could be related to other physiological mechanisms.

Another hypothesis would be that the hypothalamic-pituitary-adrenal (HPA) axis activity might be connected to the working mechanism of LF-TENS for insomnia. The existing literature has documented the bidirectional relationship between HPA axis hyperactivity and insomnia ${ }^{41,42}$, and LF-TENS can downregulate HPA axis activity since muscle relaxation that might be achieved with LF-TENS, was reported to reduce cortisol secretion ${ }^{43}$. It is well-known that cortisol acts as a marker of HPA axis function since it is released from adrenal gland in response to physical and psychological stress and its serum concentration is regulated by HPA activity ${ }^{44}$. On the basis of the aforementioned grounds, we hypothesized that serum cortisol levels would be decreased after LF-TENS treatment. As expected, we found a significantly more remarkable decrease in cortisol levels in the experimental group compared to the control group among the elderly patients, while no significant between-group difference was found in the middle-aged patients. These results support evidence that the therapeutic efficacy of LF-TENS may arise from the modulation of HPA axis activity, since this hypothesis can explain the reason why LF-TENS was more effective among older patients in our study. Unfortunately, only few studies have dealt with the effects of TENS on HPA axis activity in humans and they have shown contradictory results. Chu and colleagues have reported increased salivary cortisol levels after TENS ${ }^{45}$, while a study of the effects of TENS on the plasma concentration of cortisol failed to find a significant difference in cortisol levels ${ }^{46}$. For these reasons, further investigations 
are needed to clarify the exact physiological process underlying the clinical benefits of LF-TENS on insomnia.

To the best of our knowledge, our pilot study was the only study assessing the efficacy of LF-TENS for insomnia patients with no underlying disease, representing the response rate of $57.5 \%$ in chronic insomnia patients aged 55 years or older ${ }^{24}$. In the current study, the positive treatment response rate was $37.0 \%$ for insomnia patients aged 40 to 80 years. This relatively low response rate may be attributable to the age difference in study participants and more strict definition of positive treatment response in the current study. Moreover, we found that $42.5 \%$ of elderly insomnia patients aged over 60 years had achieved optimal treatment outcomes with LF-TENS, representing significantly better improvement of insomnia symptoms compared to those with sham treatment.

We observed the therapeutic efficacy of LF-TENS among participants over 60 years of age while no significant treatment effect was found in the middle-aged group. This result might stem from the normal sleep physiology with aging and different manifestation of insomnia symptoms in older adults. A metaanalysis has revealed that total sleep time, sleep efficiency, and slow wave sleep decreased with age in the general healthy population ${ }^{47}$. The natural shortening of sleep time in the elderly can generate anxiety about sleep duration and dysfunctional sleep habits such as spending more time in bed without gaining additional sleep time ${ }^{48}$, which may lead to poor sleep efficiency and frequent sleep fragmentation in the elderly insomnia patients ${ }^{49}$. In the present study, the myorelaxant effects of LF-TENS ${ }^{50}$ may play a pivotal role in the treatment of insomnia symptoms since the reduction of muscle tension and somatic arousal could be achieved by muscle relaxation ${ }^{51}$. This therapeutic action of LF-TENS could be more effective in the elderly patients due to their higher levels of sleep-related anxiety and stress response, compared to their younger counterparts.

The strengths of this study include the large study population consisting of insomnia patients without medical and psychiatric comorbidities which may affect the sleep quality. Moreover, our study is the first randomized-controlled trial providing some evidence for the use of LF-TENS for the treatment of insomnia among the elderly. Yet, the current study has several limitations to be considered. First, we cannot generalize our findings to severe insomnia patients requiring ongoing treatment since we excluded those with recent insomnia treatment history. However, co-existing insomnia therapies may blur the effect of LFTENS and thereby weaken the clinical implications of the study. Second, all the study participants were 40 years or older, and thus our results cannot be generalized for all ages. Finally, there were individual differences in adherence to treatment, which might affect the outcome of our analysis. But these differences could not be well controlled, and also there was no significant difference in compliance between the experimental and control group.

In conclusion, LF-TENS was safe and showed modest effects for the treatment of insomnia in elderly patients over 60 years of age. The modulation of HPA axis activity after intervention might be related to the therapeutic efficacy. Physicians may consider LF-TENS as a novel and safe therapeutic option for the management of insomnia in the elderly insomnia patients. A larger scale, highly controlled human studies 
are required to provide better insight into the therapeutic efficacy and long-term consequences of LF-TENS for the management of insomnia disorder.

\section{Methods}

\section{Study population}

The current study was conducted at two medical centers, Seoul National University Bundang Hospital and Asan Medical Center in Korea. People aged 40 to 80 years with chronic insomnia symptoms were recruited by advertisement in the local community from September 2017 to December 2019. We included those who were diagnosed with insomnia disorder based on clinical interview at the first visit, using criteria from the Diagnostic and Statistical Manual of Mental Disorders, Fifth Edition (DSM-5) ${ }^{25}$. The exclusion criteria were as follows: (1) insomnia treatment history in the recent one year; (2) sleep apnea syndrome (apneahypopnea index $\geq 15$ events/hr) or restless legs syndrome according to the diagnostic criteria of international restless legs syndrome study group (IRLSSG) ${ }^{26}$; (3) major psychiatric disorders including mood disorders, anxiety disorders, dementia, and psychotic disorders; (4) medical illness such as cardiovascular diseases, malignancy, impaired renal or hepatic function, and lung diseases; (5) head trauma or seizure disorders; (6) infectious diseases; (7) diabetes mellitus on insulin therapy; and (8) pregnancy. This trial was registered in the Clinical Research Information Service, Republic of Korea (registration number KCT0002705).

\section{LF-TENS Intervention}

As a LF-TENS device, we used CR- ${ }^{\circledR}$ medical device manufactured by Crown Medical, Inc. (Seoul, Korea). It was approved by the Korea Ministry of Food and Drug Safety and is currently commercially available for relieving muscular pain. The stimulator delivers an alternating current of $<1 \mathrm{~mA}$ (peak output voltage ranges from 0.3 to $0.64 \mathrm{~V}$ ) at a frequency of $400 \mathrm{~Hz}$, with a pulse duration of 100 microseconds. Electric current is transmitted through three transcutaneous nickel plate electrodes attached to the back and neck area to stimulate both trapezius muscles. The sham device was created to look identical to the CR-9 device, but it has no electrical stimulation function.

Eligible participants were randomly assigned in a 1:1 ratio to either the experimental group receiving an active device, or control group receiving a sham device. The random assignment was performed via the stratified permuted block randomization method, using SAS version 9.4 and both patients and investigators were blind to the intervention condition. After assignment, patients were instructed to wear the device for an hour just before bedtime, more than five days weekly, for four weeks. Although the optimal dose and duration of LF-TENS application for insomnia treatment were not established, our preliminary study demonstrated the modest efficacy and safety of CR- $9^{\circledR}$ medical device for improving chronic insomnia, using the same protocol of device use ${ }^{24}$. A recent review has also indicated that sufficient therapeutic effects could be attained by daily use of electrical stimulation for more than four weeks, with a session duration of 20 to 60 minutes ${ }^{27}$. The patients were allowed to take a rescue medication (zolpidem 5 to $10 \mathrm{mg}$ ) if they experience the aggravation of insomnia symptoms for more than five consecutive days or 
reduction of total sleep time below three hours at least one day. For verifying the safety of the device use, we have checked the occurrence of any adverse event after two and four weeks of intervention. All the adverse reactions were recorded, using codes specified in the World Health Organization adverse reaction terminology (WHOART) version 92, updated by the Uppsala monitoring center.

\section{Demographic and clinical characteristics}

At the first visit, demographic and anthropometric data were gathered, including age, sex, body-mass index (BMI), and medication use. Simultaneously, we examined the presence of any medical illness, psychiatric disorders, or sleep disorders according to the exclusion criteria. And then all the individuals underwent an overnight polysomnography (PSG) at the sleep clinic of Seoul National University Bundang Hospital for screening out those with sleep apnea syndrome (apnea-hypopnea index $\geq 15$ events $/ h$ ). An EmblaTMN7000 device (Embla, Reykjavik, Iceland) was used for PSG. Sleep stages and respiratory events were scored by PSG technicians and sleep specialists every 30 seconds epoch, according to the American Academy of Sleep Medicine (AASM) criteria ${ }^{28}$.

Before and after treatment, participants were asked to complete self-report questionnaires concerning sleep quality, psychiatric symptoms, and pain. The pre- and post-intervention evaluations were performed in the morning after overnight PSG and in the last visit after the end of intervention, respectively. As for the primary outcome measure, the Pittsburgh Sleep Quality Index (PSQI) was adopted to assess subjective sleep quality ${ }^{29}$. Since there is no established definition for clinically significant treatment response in insomnia ${ }^{30}$, we defined the positive treatment response as a decrease in PSQI score $\geq 3$ points after intervention, based on several preceding insomnia treatment studies ${ }^{30,31}$. Different from these studies, however, we did not adopt the criteria based on sleep diary data due to the unavailability of data for some participants. The secondary outcome measures were the changes in the scores of Epworth Sleepiness Scale (ESS) ${ }^{32}$, Beck Depression Inventory (BDI) ${ }^{33}$, Beck Anxiety Inventory (BAI) ${ }^{34}$, and Numerical Rating Scale for pain (NRS) ${ }^{35}$.

\section{Sleep diaries and Actigraphy}

Subjective and objective sleep parameters were assessed as secondary outcome measures. To obtain subjective sleep parameters, participants were asked to keep a sleep diary every day in the morning during the entire intervention period. The sleep diary contained items evaluating subjective sleep factors such as time in bed (TIB, the number of minutes spent in bed), sleep latency (SL, the number of minutes taken to fall asleep), wake after sleep onset (WASO, the number of minutes of wakefulness after sleep onset), TST, and sleep efficiency (SE, the ratio of TST to TIB). For examining the changes in subjective sleep quality before and after the treatment, we compared sleep parameters from sleep diaries during the pre-and postintervention actigraphic recordings. We excluded the sleep diary data from participants whose answers were obviously unreliable, for example, "TST $=0$." To confirm the adherence to the intervention protocols, the duration of daily device use was also recorded in daily sleep diaries.

For the assessment of objective sleep parameters, our study participants were requested to wear an accelerometer (wGT3X-BT, ActiGraph, LLC, Pensacola, FL, USA) on their non-dominant wrist. Actigraphic 
monitoring was performed twice, before and after treatment. The pre- and post-intervention assessments were carried out for four days just before the start of intervention and for the last four days of the intervention period, respectively. The wrist actigraphy estimated sleep-wake status by capturing and recording physical activity and information regarding TIB was obtained from the daily sleep diary. ActiLife 6 software (ActiGraph, LLC, Pensacola, FL, USA) was used to analyze raw data and calculate the following four sleep variables: SL, WASO, TST, and SE. Data of participants who made proper use of actigraphy as instructed for at least 3 days for both times were included for the analysis.

\section{Blood tests}

The pre- and post-intervention blood tests were conducted as secondary outcome measures, concurrently with the questionnaire assessments. Blood samples were drawn from the antecubital vein in the morning (between 8AM and 9AM), in the fasting state more than 8 hours. They were properly processed and transported to the testing institute (Seoul Clinical Laboratories, Seoul, South Korea). We estimated the serum levels of Interleukin 6 (IL-6), Tumor necrosis factor alpha (TNF-alpha), glucose, C-reactive protein (CRP), cortisol, and insulin.

\section{Statistical analysis}

We compared demographic and clinical characteristics between the experimental and control groups, using the independent $t$-test for continuous variables and the chi-square test for categorical variables. Paired ttest or Wilcoxon signed rank test was adopted to examine the intra-group differences between pre-and postintervention assessments including questionnaires, sleep diaries, wrist actigraphy, and blood tests. To evaluate the inter-group differences in the changes of parameters after treatment between the two study arms, we used analysis of covariance (ANCOVA) adjusted by age, sex, and baseline BMI. The exploratory analysis was performed after the patients were divided into two groups: those aged $>60$ years and those aged $\leq 60$ years. Cohen's $d$ was calculated to estimate the effect size of the intervention in each group. All statistical analyses were carried out using SPSS version 25.0 for Windows (IBM Corp., Armonk, NY, USA) and a two-tailed p-value of less than 0.05 was considered statistically significant.

\section{Ethics statement}

All the participants were informed of the purpose and procedures of the study and they provided written consent at the first visit. All study procedures were conducted in accordance with the Declaration of Helsinki and were approved by the Institutional Review Board of Seoul National University Bundang Hospital (approval number E-1705/396-001) and the Korea Food and Drug Administration (approval number 786).

\section{Declarations}

\section{Acknowledgements}

This study was supported by the Korean Health Technology R\&D Project, Ministry of Health \& Welfare, Republic of Korea (grant HI17C0203). 


\section{Author contributions}

H.C., S.C., and IY.Y. designed the study and wrote the protocol. H.J.L., J.K.H., H.C., S.C., and IY.Y. contributed to data acquisition and analysis. H.J.L. and IY.Y. wrote the first draft of the paper. IY.Y. and S.C are cocorresponding authors. All authors contributed to and have approved the final paper.

\section{Competing Interests}

The author(s) declare no competing interests.

\section{Data availability}

The datasets generated during and/or analysed during the current study are available from the corresponding author on reasonable request.

\section{References}

1. Lack, L., Miller, W. \& Turner, D. A survey of sleeping difficulties in an Australian population. Community Health Studies. 12, 200-207 (1988).

2. Zheng, W. et al. Prevalence of insomnia symptoms and their associated factors in patients treated in outpatient clinics of four general hospitals in Guangzhou, China. BMC psychiatry. 18, 232 (2018).

3. Bhaskar, S., Hemavathy, D. \& Prasad, S. Prevalence of chronic insomnia in adult patients and its correlation with medical comorbidities. Journal of family medicine and primary care. 5, 780 (2016).

4. Health, N. I. o. National Institutes of Health State of the Science Conference statement on manifestations and management of chronic insomnia in adults, June 13-15, 2005. Sleep 28, 10491057(2005).

5. Morin, C. M. et al. The natural history of insomnia: a population-based 3-year longitudinal study. Archives of internal medicine. 169, 447-453 (2009).

6. Laugsand, L. E., Vatten, L. J., Platou, C. \& Janszky, I. Insomnia and the risk of acute myocardial infarction: a population study. Circulation. 124, 2073-2081 (2011).

7. Baglioni, C. et al. Insomnia as a predictor of depression: a meta-analytic evaluation of longitudinal epidemiological studies. Journal of affective disorders. 135, 10-19 (2011).

8. Yaffe, K., Falvey, C. M. \& Hoang, T. Connections between sleep and cognition in older adults. Lancet Neurol. 13, 1017-1028 (2014).

9. Riemann, D. et al. European guideline for the diagnosis and treatment of insomnia. Journal of sleep research. 26, 675-700 (2017).

10. Riemann, D. \& Perlis, M. L. The treatments of chronic insomnia: a review of benzodiazepine receptor agonists and psychological and behavioral therapies. Sleep medicine reviews. 13, 205-214 (2009).

11. Paterniti, S., Dufouil, C. \& Alpérovitch, A. Long-term benzodiazepine use and cognitive decline in the elderly: the Epidemiology of Vascular Aging Study. Journal of clinical psychopharmacology. 22, 285293 (2002). 
12. Morin, C. M. et al. Cognitive behavioral therapy, singly and combined with medication, for persistent insomnia: a randomized controlled trial. Jama. 301, 2005-2015 (2009).

13. Cartwright, R. D. \& Weiss, M. F. The effects of electrosleep on insomnia revisited. J Nerv Ment Dis. 161, 134-137 https://doi.org/10.1097/00005053-197508000-00008 (1975).

14. Feighner, J. P., Brown, S. L. \& Olivier, J. E. Electrosleep therapy. A controlled double blind study. J Nerv Ment Dis. 157, 121-128 (1973).

15. Kirsch, D. L. \& Gilula, M. CES in the Treatment of Insomnia: A Review and Meta-analysis. Practical Pain Management. 7, 30-43 (2007).

16. Frankel, B. L., Buchbinder, R. \& Snyder, F. Ineffectiveness of electrosleep in chronic primary insomnia. Archives of General Psychiatry. 29, 563-568 (1973).

17. Lande, R. G. \& Gragnani, C. Efficacy of cranial electric stimulation for the treatment of insomnia: a randomized pilot study. Complementary therapies in medicine. 21, 8-13 (2013).

18. Shekelle, P. G. et al. Benefits and harms of cranial electrical stimulation for chronic painful conditions, depression, anxiety, and insomnia: a systematic review. Annals of internal medicine. 168, 414-421 (2018).

19. Vance, C. G., Dailey, D. L., Rakel, B. A. \& Sluka, K. A. Using TENS for pain control: the state of the evidence. Pain Manag. 4, 197-209 https://doi.org/10.2217/pmt.14.13 (2014).

20. Johnson, M. I., Paley, C. A., Howe, T. E. \& Sluka, K. A. Transcutaneous electrical nerve stimulation for acute pain. Cochrane Database Syst Rev. Cd006142, https://doi.org/10.1002/14651858.CD006142.pub3 (2015).

21. Maillefert, J. F. et al. Effects of low-frequency electrical stimulation of quadriceps and calf muscles in patients with chronic heart failure. Journal of Cardiopulmonary Rehabilitation and Prevention. 18, 277-282 (1998).

22. Bonnet, M. H. \& Arand, D. L. Hyperarousal and insomnia: state of the science. Sleep medicine reviews. 14, 9-15 (2010).

23. Gozani, S. N., Ferree, T. C., Moynihan, M. \& Kong, X. Impact of transcutaneous electrical nerve stimulation on sleep in chronic low back pain: a real-world retrospective cohort study. Journal of Pain Research. 12, 743 (2019).

24. Bang, Y. R., Jeon, H. J. \& Yoon, I. Y. Modest Effects of Low-frequency Electrical Stimulation on Patients with Chronic Insomnia in an Open Trial. Sleep Medicine Research. 10, 17-24 (2019).

25. Association, A. P. Diagnostic and statistical manual of mental disorders (DSM-5®)(American Psychiatric Pub, 2013).

26. Allen, R. P. et al. Restless legs syndrome/Willis-Ekbom disease diagnostic criteria: updated International Restless Legs Syndrome Study Group (IRLSSG) consensus criteria-history, rationale, description, and significance. Sleep medicine. 15, 860-873 (2014).

27. Kirsch, D. L. \& Nichols, F. Cranial electrotherapy stimulation for treatment of anxiety, depression, and insomnia. Psychiatric Clinics. 36, 169-176 (2013). 
28. Iber, C. J. T. \& Specification, T. The AASM manual for the scoring of sleep and associated events: Rules. (2007).

29. Buysse, D. J., Reynolds, C. F., Monk, T. H., Berman, S. R. \& Kupfer, D. J. The Pittsburgh Sleep Quality Index: a new instrument for psychiatric practice and research. Psychiatry res. 28, 193-213 (1989).

30. Buysse, D. J., Ancoli-Israel, S., Edinger, J. D., Lichstein, K. L. \& Morin, C. M. Recommendations for a standard research assessment of insomnia. Sleep. 29, 1155-1173 (2006).

31. Troxel, W. M., Conrad, T. S., Germain, A. \& Buysse, D. J. Predictors of treatment response to brief behavioral treatment of insomnia (BBTI) in older adults. Journal of Clinical Sleep Medicine. 9, 12811289 (2013).

32. Johns, M. W. A new method for measuring daytime sleepiness: the Epworth sleepiness scale. sleep 14, 540-545(1991).

33. Beck, A. T., Steer, R. A. \& Brown, G. K. Beck depression inventory-II. San Antonio. 78, 490-498 (1996).

34. Steer, R. A. \& Beck, A. T.Beck Anxiety Inventory. (1997).

35. Hawker, G. A., Mian, S., Kendzerska, T. \& French, M. Measures of adult pain: Visual analog scale for pain (vas pain), numeric rating scale for pain (nrs pain), mcgill pain questionnaire (mpq), short-form mcgill pain questionnaire (sf-mpq), chronic pain grade scale (cpgs), short form-36 bodily pain scale (sf-36 bps), and measure of intermittent and constant osteoarthritis pain (icoap). Arthritis care \& research. 63, S240-S252 (2011).

36. Gavid, M., Mayaud, A., Timochenko, A., Asanau, A. \& Prades, J. Topographical and functional anatomy of trapezius muscle innervation by spinal accessory nerve and $\mathrm{C} 2$ to $\mathrm{C} 4$ nerves of cervical plexus. Surgical and Radiologic Anatomy. 38, 917-922 (2016).

37. Steingrímsdóttir, Ã. A., Vøllestad, N. K. \& Knardahl, S. A prospective study of the relationship between musculoskeletal or psychological complaints and muscular responses to standardized cognitive and motor tasks in a working population. European Journal of Pain. 9, 311-324 (2005).

38. Sjörs, A., Larsson, B., Persson, A. L. \& Gerdle, B. An increased response to experimental muscle pain is related to psychological status in women with chronic non-traumatic neck-shoulder pain. $B M C$ musculoskeletal disorders. 12, 230 (2011).

39. Mathias, J., Cant, M. \& Burke, A. Sleep disturbances and sleep disorders in adults living with chronic pain: a meta-analysis. Sleep medicine. 52, 198-210 (2018).

40. Hester, J. \& Tang, N. K. Insomnia co-occurring with chronic pain: clinical features, interaction, assessments and possible interventions. Reviews in pain. 2, 2-7 (2008).

41. Buckley, T. M. \& Schatzberg, A. F. On the interactions of the hypothalamic-pituitary-adrenal (HPA) axis and sleep: normal HPA axis activity and circadian rhythm, exemplary sleep disorders. The Journal of Clinical Endocrinology \& Metabolism. 90, 3106-3114 (2005).

42. Vgontzas, A. N. et al. Chronic insomnia is associated with nyctohemeral activation of the hypothalamic-pituitary-adrenal axis: clinical implications. The Journal of Clinical Endocrinology \& Metabolism. 86, 3787-3794 (2001). 
43. Chellew, K., Evans, P., Fornes-Vives, J., Perez, G. \& Garcia-Banda, G. The effect of progressive muscle relaxation on daily cortisol secretion. Stress. 18, 538-544 (2015).

44. Miller, D. B. \& O'Callaghan, J. P. Neuroendocrine aspects of the response to stress. Metabolism-Clinical and Experimental. 51, 5-10 (2002).

45. Chu, H., Li, M. H., Juan, S. H. \& Chiou, W. Y. Effects of transcutaneous electrical nerve stimulation on motion sickness induced by rotary chair: a crossover study. The Journal of Alternative and Complementary Medicine. 18, 494-500 (2012).

46. Rodriguez, E. et al. Effects of transcutaneous nerve stimulation on the plasma and CSF concentrations of beta-endorphin and the plasma concentrations of $\mathrm{ACTH}$, cortisol and prolactin in hysterectomized women with postoperative pain. Revista espanola de anestesiologia y reanimacion. 39, 6-9 (1992).

47. Ohayon, M. M., Carskadon, M. A., Guilleminault, C. \& Vitiello, M. V. Meta-analysis of quantitative sleep parameters from childhood to old age in healthy individuals: developing normative sleep values across the human lifespan. Sleep. 27, 1255-1273 (2004).

48. Hirshkowitz, M., Moore, C. A., Hamilton, C. 3rd, Rando, K. C. \& Karacan, I. Polysomnography of adults and elderly: sleep architecture, respiration, and leg movement. Journal of Clinical Neurophysiology: Official Publication of the American Electroencephalographic Society. 9, 56-62 (1992).

49. Pillai, V., Roth, T. \& Drake, C. L. The nature of stable insomnia phenotypes. Sleep. 38, 127-138 (2015).

50. Esclassan, R. et al. Optimal duration of ultra low frequency-transcutaneous electrical nerve stimulation (ULF-TENS) therapy for muscular relaxation in neuromuscular occlusion: A preliminary clinical study. Cranio. 35, 175-179 https://doi.org/10.1080/08869634.2016.1171479 (2017).

51. Taylor, D. J. \& Roane, B. M. J. J. o. c. p. Treatment of insomnia in adults and children: a practicefriendly. review of research. $66,1137-1147$ (2010).

\section{Figures}




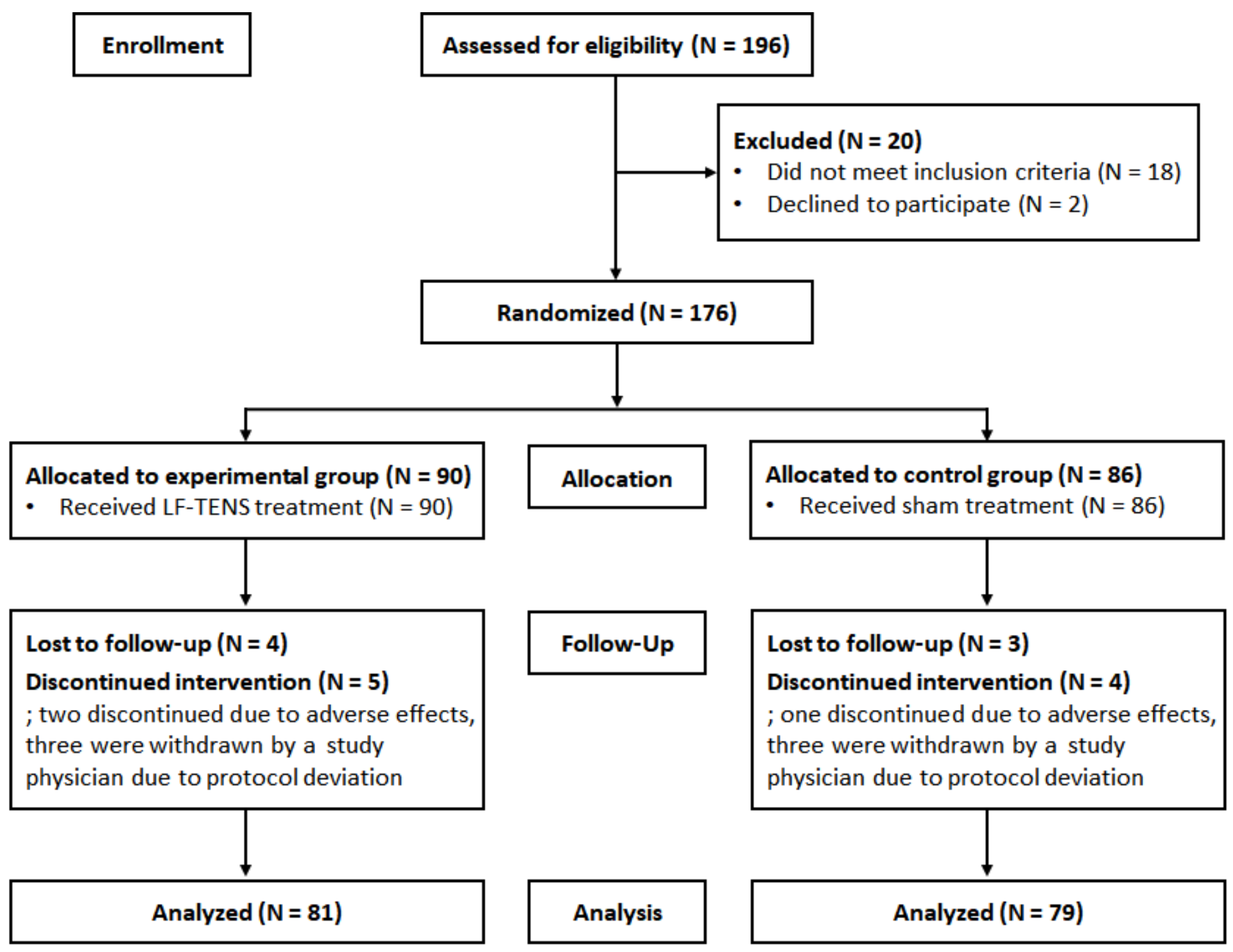

Figure 1

Flowchart of study participants 
A

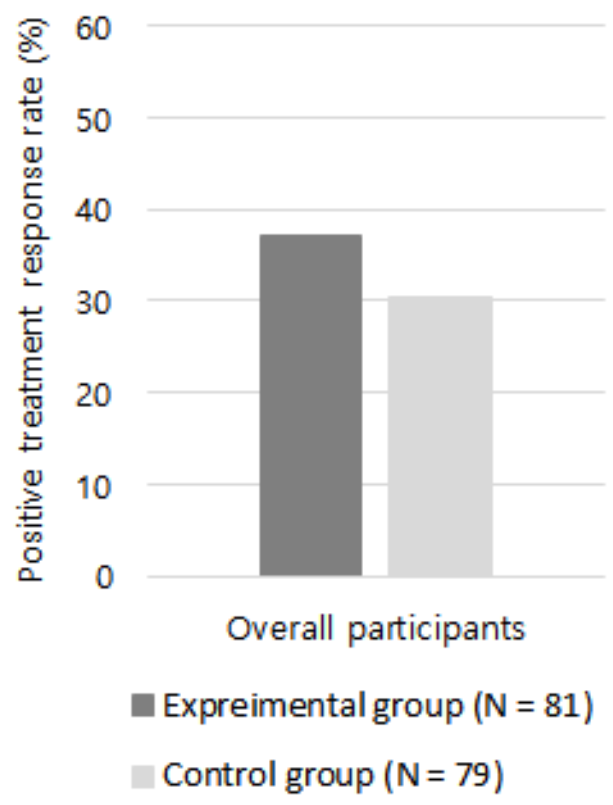

B

60

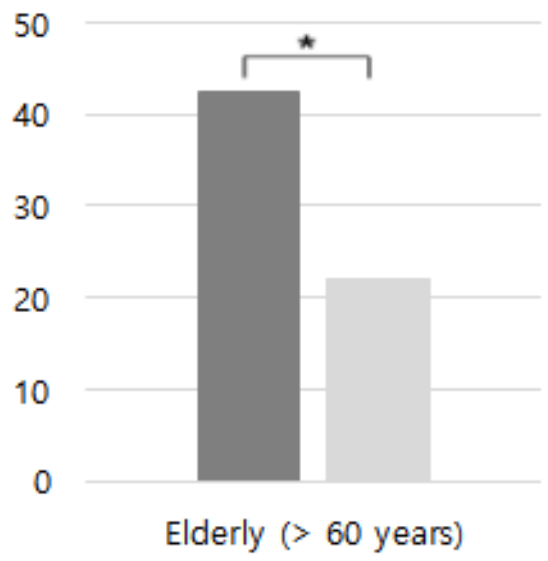

Expreimental group $(\mathrm{N}=40)$

Control group $(\mathrm{N}=41)$
C

60

50

40

30

20

10

0

Middle-aged (40-60 years)

Expreimental group $(\mathrm{N}=41)$

Control group $(\mathrm{N}=38)$

\section{Figure 2}

The positive treatment response rate in the experimental and control group among overall participants, elderly patients (aged $>60$ years), and middle-aged patients (aged 40-60 years) 
Active treatment

\section{2}

10

Positive response

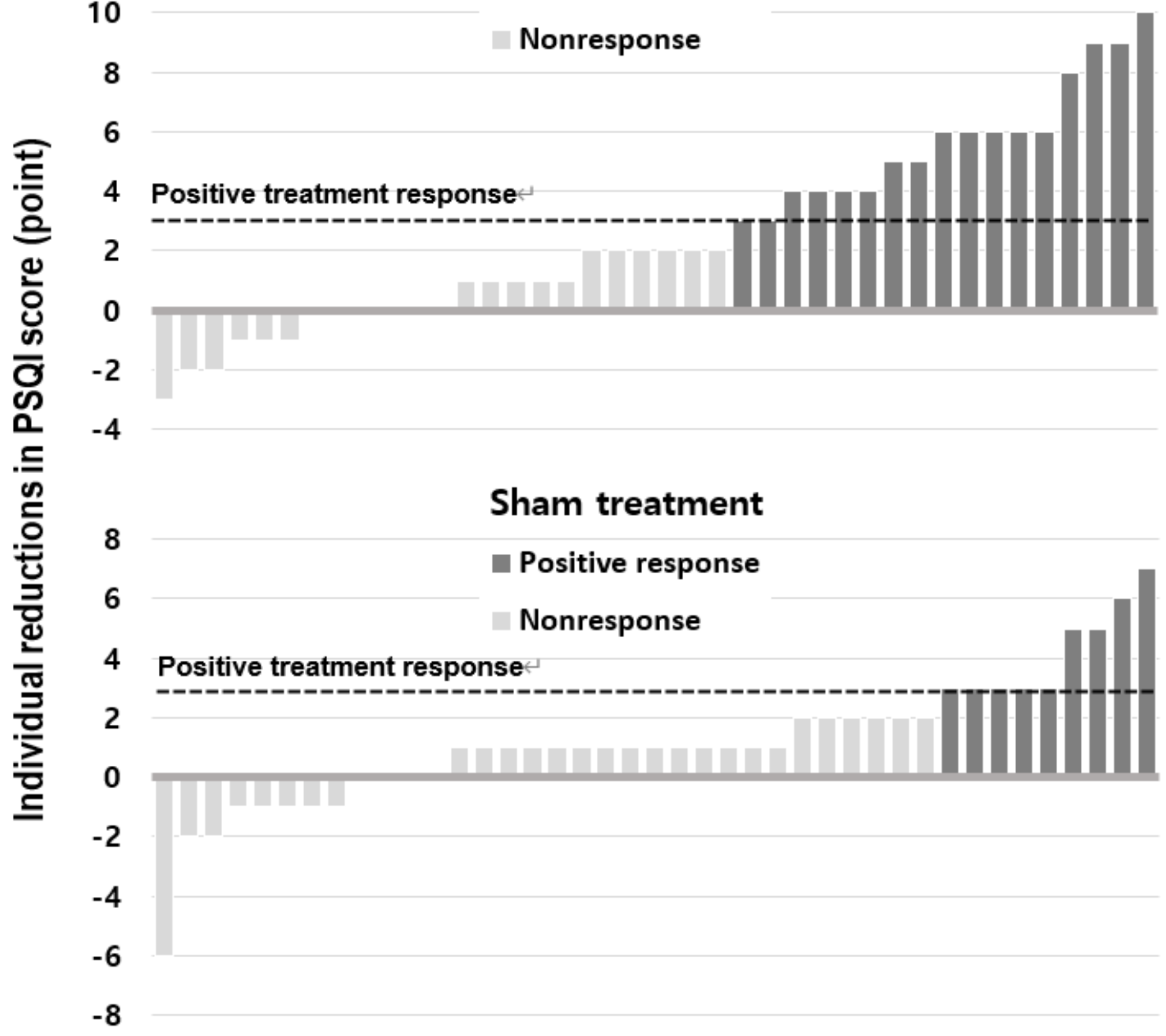

Figure 3

Individual distribution of elderly patients aged $>60$ years with and without positive treatment response according to the reduction in PSQI score after the active and sham treatment 